\title{
Spectrum Imaging and Scatter Diagrams for the Nanoscale Morphology Characterization of Hydrated Polymers
}

\author{
Alioscka Sousa and Matthew Libera \\ Dept. of Chemical Engineering, Stevens Institute of Technology, Hoboken, NJ 07030, USA
}

Spectrum imaging using electron energy-loss spectroscopy (EELS) in the cryo-STEM is emerging as an important technique for spatially resolved compositional measurements in hydrated soft materials $[1,2,3]$. Here we study the early nanoscale morphological changes that occur in a PDTEPEG bioresorbable copolymer [4] during hydrolytic degradation and erosion in water. PDTE is a tyrosine-derived hydrophobic polycarbonate. The length scales characterizing the early morphological changes can be very small, and thus they challenge the limits of detection and spatial resolution of the spectrum-imaging approach. Previous works have reported a spatial resolution in the study of hydrated soft materials in the range of 80-200 $\mathrm{nm}$. Trying to further increase resolution by decreasing the incident probe diameter, however, must be accompanied by a decrease in the number of electrons that hit the specimen to keep the electron dose below the threshold for damage in this sensitive class of materials. As a result, spectrum images can be significantly noisy, and their interpretation is not always possible from a simple visual inspection. Here we use scatter diagrams together with noise simulations to interpret water and PEG compositional maps that we collect with a spatial resolution of $15 \mathrm{~nm}$, a factor of five or more better than previously achieved.

Scatter diagrams are composition-composition images where the intensity at each pixel corresponds to the frequency with which a given composition appears [3,5]. Consider a threecomponent system consisting of perfectly mixed water, hydrophilic $\left(\mathrm{H}_{1}\right)$, and hydrophobic $\left(\mathrm{H}_{2}\right)$ polymers. Fig.1a shows the water-H1 scatter diagram derived from the water and H1 compositional maps. Since the system is homogeneous, the diagram should have a single point at a specific $\left(\mathrm{X}_{1}, \mathrm{Y}_{2}\right)$ coordinate. However, because of noise the single composition appears as a cluster of points spread around $\left(\mathrm{X}_{1}, \mathrm{Y}_{2}\right)$. Next, consider the same water, $\mathrm{H}_{1}$, and $\mathrm{H}_{2}$ system now with projected composition varying pixel by pixel in the spectrum image. Fig.1b shows the scatter diagram generated from the compositional maps. To quantitatively interpret this diagram we need to know the noise cluster, i.e., the size and shape of the cluster of points around a specific composition that is solely due to noise. This cluster depends on factors such as the shape of the reference spectra used for multiple-least-squares fitting, electron dose, and probe size, and elsewhere we describe how to calculate the noise cluster for any set of experimental conditions [3]. The ellipse shown in Fig.1b encompasses $99 \%$ of the calculated noise cluster centered at a specific $\left(\mathrm{X}_{3}, \mathrm{Y}_{4}\right)$ coordinate. Thus, all the points outside of the ellipse are not associated with the composition at $\left(\mathrm{X}_{3}, \mathrm{Y}_{4}\right)$, and by tracing back these points to the maps we unambiguously find the pixels in the maps that are statistically significant. We point out that many experimental points inside the ellipse in Fig. $1 \mathrm{~b}$ are not noise. However, we can not trace these points back to the maps because inside the ellipse we can not separate them from those points that are noise.

Fig.2a shows 15-nm resolution water and PEG maps from a PDTE-PEG copolymer that absorbed water for two days where degradation is negligible. The scatter diagram derived from the maps together with the calculated noise cluster is shown in Fig.2b, and, because the noise cluster is well superimposed on the experimental points, we conclude that water and PEG are initially homogeneously distributed in the specimen at the spatial resolution of $15 \mathrm{~nm}$. Water and PEG maps after aging the PDTE-PEG copolymer in water for 5 months is shown in Fig.2c. The scatter 
diagram with associated noise cluster (Fig.2d) indicates again that water and PEG are homogeneously distributed. Thus, despite the formation of highly water-soluble PEG-rich fragments from the copolymer degradation, these fragments do not significantly erode from the matrix and consequently cause no detectable morphological changes. Lack of erosion can be understood adopting the hypothesis that after 5 months no percolated microstructure [6] has developed through which PEG-rich fragments can diffuse. Fig.2e shows now water and PEG maps after aging the specimen for 12 months in water. The associated scatter diagram (Fig.2f) shows a number of experimental points falling outside of the noise cluster, and this unmistakably indicates that now water and PEG are distributed heterogeneously within the specimen. More specifically, from the PEG map we observe erosion within circular as well as elongated specimen regions with characteristic length scales of 50-100 nm, and from the water map we see that within the PEGeroded regions there is a localized increase in water uptake. From these results we conclude that PEG leaves the copolymer bulk preferentially to PDTE through a percolated microstructure where the characteristic length scale of the percolation is $50-100 \mathrm{~nm}$. The ability to study the dynamic hydrated morphology of bioresorbable polymers quantitatively and at nano length scales with EELS in the cryo-STEM can open new opportunities for controlling polymer erosion based on polymer morphology rather than simply on polymer chemistry.

[1] Sun S., Shi S., Hunt J., and Leapman R. J. Microsc. 177 (1995) 18-30.

[2] Terryn C. et al. Eur. Biophys. J. 33 (2004) 321-327.

[3] Sousa A., Aitouchen A., and Libera M. Ultramicroscopy 106 (2006) 130-145.

[4] Yu C. and Kohn J. Biomaterials 20 (1999) 253-264

[5] Grogger W., Hofer F., and Kothleitner G. Micron 29 (1998) 43-51.

[6] Göpferich A. Macromolecules 30 (1997) 2598-2604.

[7] This research has been supported through RESBIO (NIH NIBIB Grant), DOD DURIP, and the NSF.
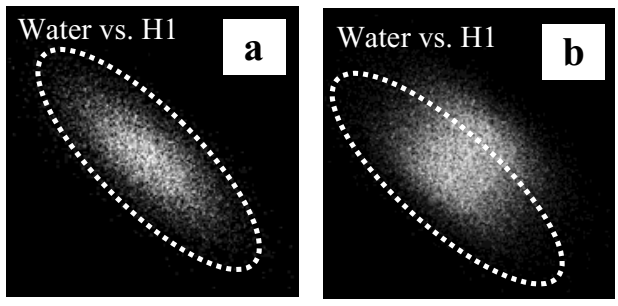

Fig.1. a) Scatter diagram from water and hydrophilic-

polymer maps assuming a hypothetical hydrated hydrophilic(H1)/hydrophobic polymeric specimen with all the components perfectly mixed. b) Scatter diagram assuming a heterogeneous spatial distribution of the components of the water-hydrophilic-hydrophobic specimen.
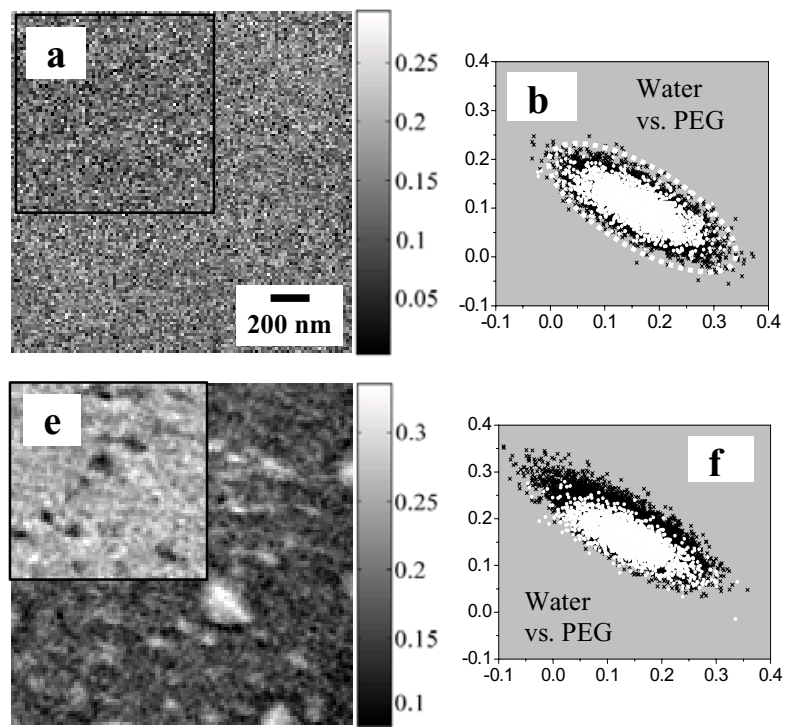
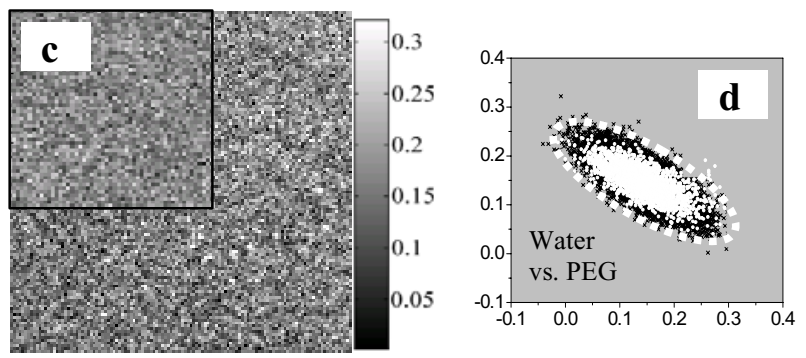

Fig.2. a) Water and PEG (inset) maps after 2 days in water. b) Scatter diagram from a) with noise cluster. c) Water and PEG maps after 5 months aging in water d) Scatter diagram from c) with noise cluster. e) Water and PEG (inset) maps after 12 months aging in water. f) Scatter diagram from e) with noise cluster. In the scatter diagrams the black points are the experimental points derived from the maps and the white points the calculated noise cluster. 